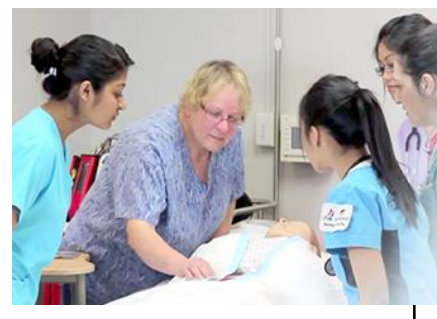

\title{
International Journal of Midwifery and Nursing Practice
}

E-ISSN: 2663-0435 P-ISSN: 2663-0427 www.nursingpractice.net IJMNP 2021; 4(1): 12-16 Received: 10-11-2020 Accepted: 15-12-2020

\section{Malathi D}

Ph.D., Scholar, Rani Meyyammai College of Nursing, Annamalai University, Chidambaram,

Tamil Nadu, India

\section{Dr. Gandhimathi M} Principal, Rani Meyyammai College of Nursing, Annamalai University, Chidambaram, Tamil Nadu, India

Dr. A Geethalakshmi Registrar, Institute of Social Obstetrics, Kasturba Gandhi Hospital for Women and Children, Chennai, Tamil Nadu, India
Corresponding Author: Malathi D

Ph.D., Scholar, Rani Meyyammai College of Nursing, Annamalai

University, Chidambaram, Tamil Nadu, India

\section{Effect of foot reflexology on fatigue and coping during first stage of labour among Primi parturient mothers in Kasturba Gandhi hospital for women and Children, Chennai}

\author{
Malathi D, Dr. Gandhimathi M and Dr. A Geethalakshmi
}

DOI: https://doi.org/10.33545/26630427.2021.v4.i1a.70

\begin{abstract}
Labour is the process by which the fetus and placenta are expelled from the uterus and the vagina into the external environment (Baskar, 2015). Successful labour depends on four integrated concepts as Passage, Passenger, Power, and Psychological outlook (Pillitteri \& Pillitteri, 2010). The onset of labour is characterized by true labour pain, show, Dilatation of cervix and formation of the bag of fore waters (Baskar, 2015). The objectives of this study were to: (1) assess the level of fatigue and coping during the first stage of labour among primi parturient mothers in the intervention group and control group (2) find the effect of foot reflexology on fatigue during the first stage of labour among primi parturient mothers in the intervention group and control group, and (3) determine the effect of foot reflexology on coping during the first stage of labour among primi parturient mothers. The study was conducted among 30 primi parturient mothers in Kasturba Gandhi Hospital for Women and Children, Chennai District, Tamil Nadu. The study finding revealed that in post-test 1 the mean fatigue score in the intervention group was 61.00 which is less than the control group 85.33 (mean difference 24.33) which supports that foot reflexology is effective in fatigue reduction $(P<0.05)$. In post-test 2 the mean fatigue score in the intervention group was 48.80 and the control group was 91.86 (mean difference 43.06) which was significant at $P<0.05$. In post-test 1 the mean coping score in the intervention group was 156.86 which is more than the control group 128.20 (mean difference 28.66), which revealed that foot reflexology is effective in promoting coping at $P<0.05$. Similarly, in post-test 2 the mean coping score in the intervention group and control group was 168.20 and 133.46 respectively (mean difference 34.74), which was significantly higher than the control group at $P<0.05$. The study concludes that foot reflexology is effective to reduce fatigue and promoting coping during the first stage of labour among primi parturient mothers.
\end{abstract}

Keywords: Foot reflexology, primi parturient mothers, maternal parameters, fatigue, coping

\section{Introduction}

Fatigue reduces a women's ability to tolerate pain and to use coping skills. She may be unable to focus on relaxation and breathing techniques that would help her to tolerate labour. Many women sleep poorly during the last weeks of pregnancy. However, fatigue is a common phenomenon throughout pregnancy until labour which increases the labour process (Ebrahimzadeh et al., 2012) ${ }^{[3]}$.

Reflexology is a holistic healing technique that aims to treat the individual as an entity, incorporating the body, mind, and spirit. It is the specific pressure technique that targets precise reflex points on the feet, based on the premise in reflex areas on the feet correspond with all other body parts (Synder Lindquist, 2006) ${ }^{[9]}$. Vennells (2001) articulates the word reflex as stimulus or reaction in the form of an increase, decrease, or rebalance of a particular physical, mental or emotional function in the body (Synder Lindquist, 2006) ${ }^{[9]}$.

Many factors contribute to the client's emotional response to birth. Lack of knowledge, fear of pain, personal or family stress, lack of labour support from a significant other, degree of self-confidence, feelings of loss of control, negative attitudes about birth, cultural background, and concerns for personal safety can all serve to block labor's progress (Goodman et al., 2004; Turley, 2004). The birth environment can influence the labour as well (Hodnett et al; 2005) (Orsan, 2008). Moreover, the maternal psychosocial responses vary based on previous coping strategies and associated events. Early in labour, the client may be apprehensive, yet excited. As labour progress and contractions increase in frequency and intensity, the women tend to focus on the work at hand. As pain increases so, many 
discouragements and fatigue, decreasing the client's ability to effectively tolerate contractions (Simpson \& Creehan, 2001). For example, during the active phase, the client experiences growing discomfort, including pressure on the bladder and rectum. Strategies she used earlier in labour may be less effective when dealing with more intense sensations. The client may respond to pain with screaming or crying. Fatigue and exhaustion may further compound these feelings. As a result, the client may develop a fear that she will completely lose control (Orsan, 2008).

Ebrahimzadeh et al., (2012) [3] was conducted a crosssectional study on the correlation between maternal fatigue and uterine contraction pattern in the active phase of labour among 100 primiparous women in Iran. The study found that, a significant relationship between maternal fatigue and uterine contraction pattern. It was concluded that maternal fatigue changes the uterine contraction pattern.

Many study findings reveal that there is a change in maternal physiological parameters during the first stage of labour among primi parturient mothers after foot reflexology. Hence, the researcher felt the need of helping the mothers and need to assess the fatigue and coping during the first stage of labour and helps them to reduce fatigue and to improve coping abilities during the first stage of labour using non-pharmacological methods.

\section{Aim and Objectives}

The present study is aimed to assess the level of fatigue and coping among primi parturient mothers during the first stage of labour in Chennai, Tamil Nadu. The objectives of this study were to: (1) assess the level of fatigue and coping during the first stage of labour among primi parturient mothers (2) find the effect of foot reflexology on fatigue during the first stage of labour among primi parturient mothers, and (3) determine the effect of foot reflexology on coping during the first stage of labour among primi parturient mothers.

\section{Materials and Methods}

A quasi-experimental non-randomized control group design was applied to find the effect of foot reflexology on fatigue and coping during the first stage of labour among primi parturient mothers. The study was conducted during October 2020 among 30 primi parturient mothers during the first stage of labour admitted in the labour room of Kasturba Gandhi Hospital for Women and Children, Chennai. Primi parturient mothers who have completed 37 weeks of gestation, with a singleton pregnancy, with vertex presentation, and who are in the latent phase were included in the study. Purposive sampling technique was used to select the subjects and the first 15 were included in the control group and another 15 were for the intervention group to avoid contamination.

The present study was conducted after getting approval from Madras Medical College Institutional Ethical Committee. Primi parturient mothers were enrolled after getting informed consent. The researcher explained about the intervention and data were collected from Primi parturient mothers during the first stage of labour was assessed by using tools like Socio-demographic proforma, Clinical variables, Fatigue during labour was assessed by Fatigue continuum scale (Pugh et al., 1999) ${ }^{[8]}$, and Coping was assessed by Modified Alice Chacko Non-coping response scale (Esther John, 2009) ${ }^{[4]}$. Internal consistency was adopted to assess the reliability of the fatigue continuum scale and Modified Chacko Alice non-coping response scale. Tools were assessed by Cronbach's Alpha based on the pilot study and the reliability scores were 0.89 and 0.83 respectively which shows high reliability.

Pre-test fatigue and coping were assessed at 1-3 cm of cervical dilatation in both groups. Further, foot reflexology was administered to the mothers in the intervention group at $4-5 \mathrm{~cm}$ of cervical dilatation for 20 minutes and the second session of foot reflexology was administered at 6-7 cm of cervical dilatation for 20 minutes. After each intervention post-test, and post-test 2 fatigue and the coping level were assessed. However, mothers in the control group received routine care and post-test 1 was assessed after 20 minutes of $4-5 \mathrm{~cm}$ of cervical dilatation and post-test 2 was assessed after 20 minutes of $6-7 \mathrm{~cm}$ of cervical dilatation respectively. Data analysis in terms of descriptive and inferential statistics was performed using SPSS v.20.

\section{Result}

The demographic data shows that the age of primi parturient mothers ranges between 18-34 years, about $40 \%$ of mothers in the intervention group belong to $18-21$ years and $40 \%$ of mothers in the control group were belongs to 22-25 years of age group. Regarding education, mothers in the intervention group have $27 \%$ of secondary education and degree and above, whereas mothers in the control group have $33 \%$ of primary education. In religion, the majority $87 \%$ of mothers were Hindus in both the intervention group and control group. Regarding family income, about $60 \%$ of mothers in the intervention group and $80 \%$ of mothers in the control group have $<10,000$ rupees per month.

Regarding the type of family, around $60 \%$ of mothers in the intervention group belong to a joint family and on contrary, $67 \%$ of mothers in the control group belong to the nuclear family. But, in both groups about $67 \%$ of mothers were from the urban community. In both the intervention and control group, the major proportion had a nonconsanguineous marriage which is $87 \%$ and $93 \%$ respectively. In the duration of married life, $60 \%$ of mothers in the intervention group and $53 \%$ of mothers in the control group reported duration of the marriage is less than one year. The majority $80 \%$ of mothers in the intervention group and $73 \%$ of mothers in the control group has opinioned as the mother is the support person. Remarkably, in both the groups, all of them (100\%) were opinioned as leading satisfactory marital relationship, pregnancy is planned, attended antenatal counselling, registered their pregnancy and not practicing complementary therapy.

The clinical variables exhibit that, the mothers of both groups were between 37-40 weeks of gestation. 67\% of mothers in the intervention group and control group had 7-9 antenatal visits. The mean scores of Hemoglobin, Height, Weight, and BMI in the intervention group were 11.29, 153.86, 64.00, and 26.91 respectively. And the mean Hemoglobin, Height, Weight, and BMI in the control group were $11.20,153.93,61.06$, and 25.82 respectively. Data also shows that majority of mothers in the intervention group $73 \%$ and $93 \%$ in the control group had induction of labour. Similarly, augmentation of labour was done for $80 \%$ of mothers in the intervention group and all mothers (100\%) in the control group. 


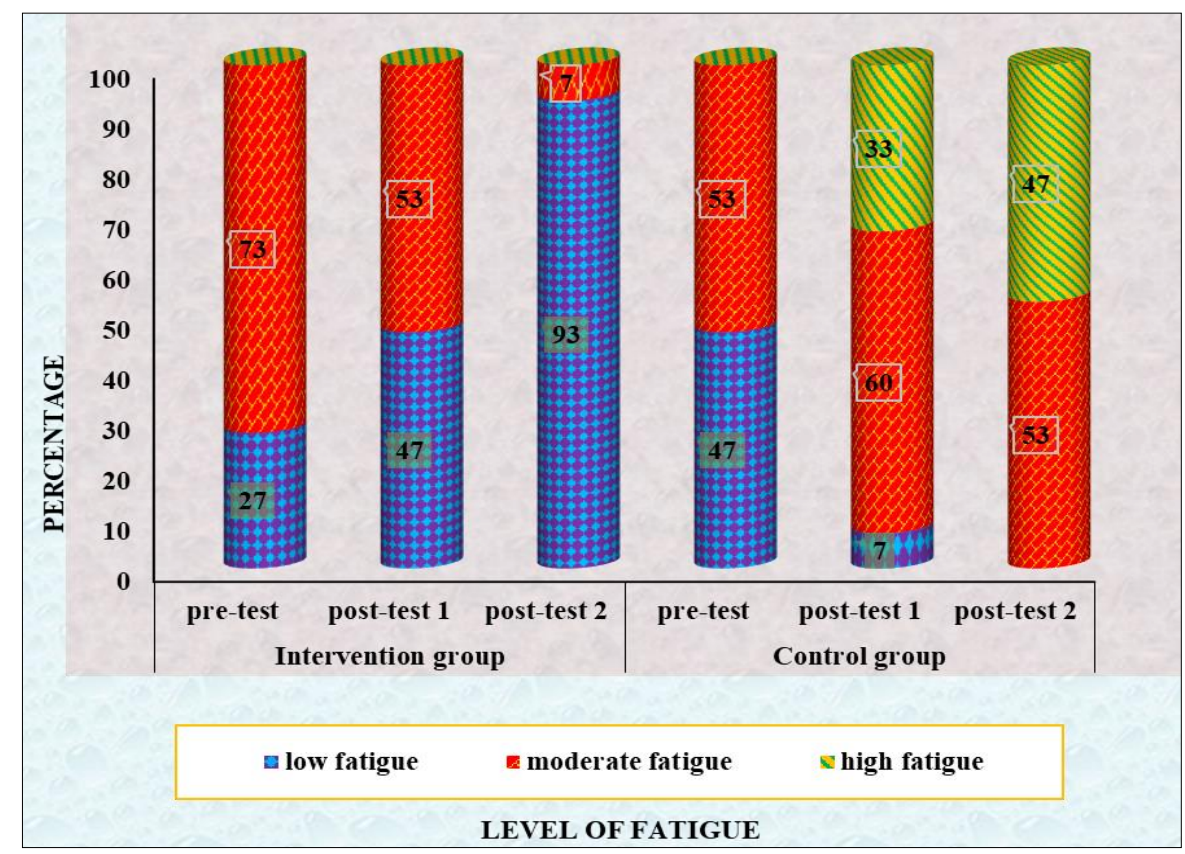

Fig 1: Percentage distribution of fatigue among intervention and control group

The level of fatigue during pre-test in the intervention group shows that the majority $73 \%$ of mothers had moderate fatigue and $27 \%$ had low fatigue, in the control group $53 \%$ reported moderate fatigue and about $47 \%$ had low fatigue. While in post-test 1 , in the intervention group, $53 \%$ of mothers had moderate fatigue and about $47 \%$ of mothers had low fatigue, whereas in the control group $60 \%$ of mothers had moderate fatigue, $33 \%$ of mothers had high fatigue level and only $7 \%$ had low fatigue. Followed by in post-test 2, 93\% of mothers had low fatigue and only $7 \%$ has moderate fatigue in the intervention group compared to $53 \%$ had moderate fatigue and $47 \%$ had high fatigue among the control group (figure 1).

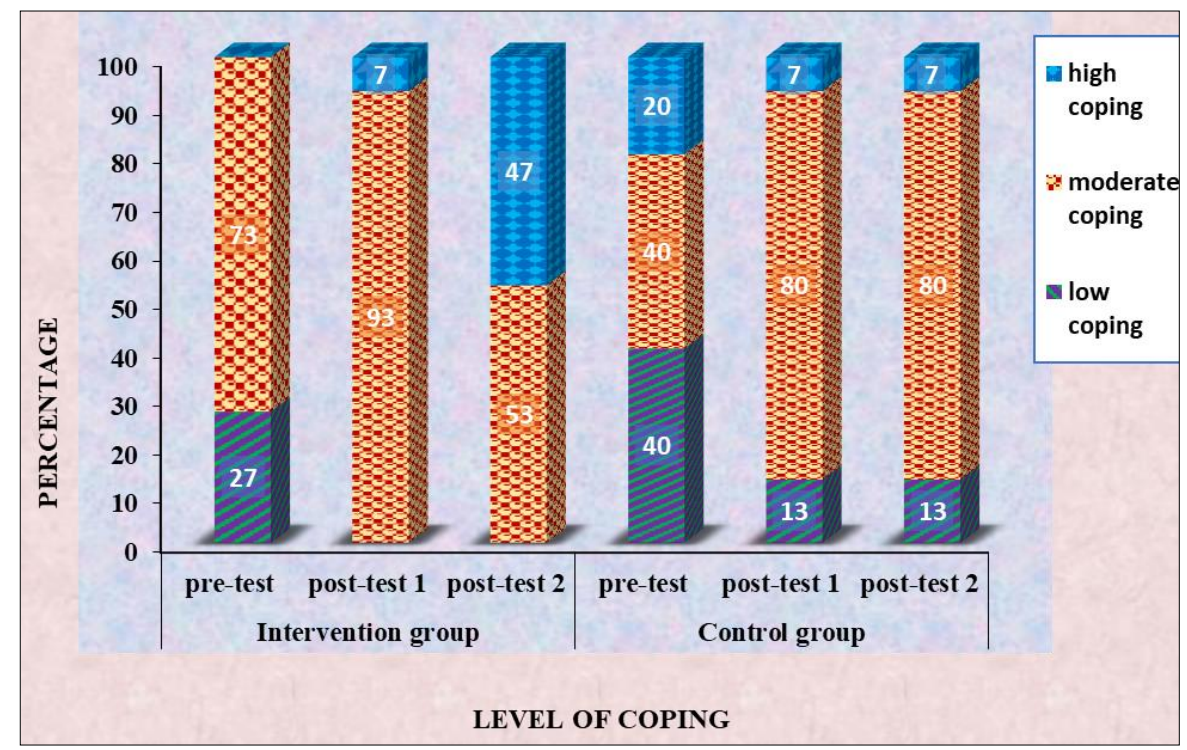

Fig 2: Percentage distribution of coping among intervention and control group

The level of coping during the first stage of labour during the pre-test revealed a marked difference in the intervention group in each stage. In the pre-test majority $73 \%$ of mothers had moderate coping, and the remaining $27 \%$ of mothers had low coping. Followed by, during post-test 1 majority $93 \%$ of mothers had moderate coping and about $7 \%$ of mothers exhibited high coping level. During post-test 2, about $53 \%$ of mothers had moderate coping and $47 \%$ of mothers reported high coping. On the other hand, mothers in the control group did not exhibit a significant change in coping. The findings showed during the pre-test about equal proportion $(40 \%)$ had moderate and low coping and only $20 \%$ had high coping. Followed by, during post-test 1 and 2 majority of mothers $80 \%$ had moderate coping, $13 \%$ had low coping, and at least $7 \%$ had high coping. However, it shows that foot reflexology has a significant change in coping level (figure 2). 
Table 1: Comparison between pre-test, post-test 1 and post-test 2 fatigue scores in intervention group and control group

\begin{tabular}{|c|c|c|c|c|c|c|c|}
\hline \multirow{2}{*}{ Fatigue level } & \multicolumn{2}{|c|}{ Intervention group $(\mathrm{n}=15)$} & \multicolumn{2}{|c|}{ Control group $(n=15)$} & \multirow{2}{*}{ Mean difference } & \multirow{2}{*}{ t-value } & \multirow{2}{*}{ p-value } \\
\hline & Mean & SD & Mean & SD & & & \\
\hline Pre-test & 66.53 & 6.523 & 64.60 & 8.508 & 1.93 & 0.698 & .491 \\
\hline Post-test1 & 61.00 & 8.254 & 85.33 & 10.223 & 24.33 & $7.172^{*}$ & $<0.05$ \\
\hline Post-test2 & 48.80 & 7.062 & 91.86 & 10.662 & 43.06 & $13.041 *$ & $<0.05$ \\
\hline
\end{tabular}

*Significance at $P<0.05, \mathrm{~N}=30$

The effect of foot reflexology in the first stage of labour in terms of fatigue shows the pre-test mean score in the intervention group was 66.53 and the control group was 64.60 (mean difference 1.93). Thus, no significant mean difference in pre-test fatigue scores among both the groups $(p>0.05)$. Further in post-test 1 , the mean fatigue score in the intervention group was 61.00 which is less than the control group 85.33 (mean difference 24.33). The present study revealed that foot reflexology is effective in fatigue reduction in terms of significant mean difference $P<0.05$. Similarly, in post-test 2 the mean fatigue score in the intervention group and control group was 48.80 and 91.86 respectively (mean difference 43.06), which revealed that the fatigue score in the intervention group was significantly lower than the control group at $P<0.05$ (table 1).

Table 2: Comparison between pre-test, post-test 1 and post-test 2 coping scores in experimental group and control group

\begin{tabular}{|c|c|c|c|c|c|c|c|}
\hline \multirow{2}{*}{ Coping level } & \multicolumn{2}{|c|}{ Intervention group $(n=15)$} & \multicolumn{2}{|c|}{ Control group $(n=15)$} & \multirow{2}{*}{ Mean difference } & \multirow{2}{*}{ t-value } & \multirow{2}{*}{ p-value } \\
\hline & Mean & SD & Mean & SD & & & \\
\hline Pre-test & 125.86 & 23.945 & 117.73 & 35.053 & 8.13 & 0.742 & .464 \\
\hline Post-test1 & 156.86 & 17.995 & 128.20 & 25.484 & 28.66 & $3.559^{*}$ & $<0.05$ \\
\hline Post-test 2 & 168.20 & 23.827 & 133.46 & 25.731 & 34.73 & $3.836^{*}$ & $<0.05$ \\
\hline
\end{tabular}

*Significance at $P<0.05, \mathrm{~N}=30$

The pre-test mean score of the intervention and control group was 125.86 and 117.73 respectively (mean difference 8.13), which revealed that there is no significant mean difference in pre-test coping level among both groups $(\mathrm{p}>0.05)$. Further in post-test 1 , the mean coping score in the intervention group was 156.86 was more than the control group 128.20 (mean difference 28.66), which revealed that foot reflexology is effective in improving coping in terms of significant mean difference $(P<0.01)$. Similarly, in post-test 2 the mean coping score in the intervention group and control group was 168.20 and 133.46 respectively (mean difference 34.73 ). Hence it revealed that the coping score of the intervention group was significantly higher than the control group at $P<0.05$ (table 2).

\section{Discussion}

The present study was conducted among 30 primi parturient mother who have completed 37 weeks of gestation, with a singleton pregnancy, with vertex presentation, and who is in the latent phase. A purposive sampling technique was used to select the study subjects. The first 15 were included in the control group and another 15 in the intervention group. The findings show that in post-test 1 the mean fatigue score in the intervention group was 61.00 which is less than the control group 85.33 which supports that foot reflexology is effective in fatigue reduction $(P<0.05)$. In post-test 2 the mean fatigue score in the intervention group was 48.80 and the control group was 91.86 which was significant at $P<0.05$. Likewise, the effect of foot reflexology on coping shows that, in post-test 1 the mean coping score in the intervention group was 156.86 which is more than the control group 128.20 which revealed that foot reflexology is effective in promoting coping at $P<0.05$. Similarly, in posttest 2 the mean coping score in the intervention group and control group was 168.20 and 133.46 respectively, which was significantly higher than the control group at $P<0.05$. The study concludes that foot reflexology is effective to reduce fatigue and promoting coping during the first stage of labour among primi parturient mothers.
The present study findings were supported by an experimental study on the effectiveness of foot reflexology upon labour pain and coping during the first stage of labour in primigravid women at Chennai. The result shows that the mean coping score was improved after foot reflexology (4.3) in the intervention group compared to the control group (3.3). The study concludes that more than $90 \%$ of study participants were highly satisfied thus foot reflexology was found effective (Anchana C K, 2013) ${ }^{[1]}$.

\section{Conclusion}

To conclude Foot reflexology is effective, the present study was carried out with 30 primi parturient mothers. Mothers in the intervention group were administered foot reflexology was compared with the mothers in the control group with routine care. The findings were revealed that Foot reflexology is effective in the reduction of fatigue and promotion of coping. It is statistically significant during the first stage of labour among primi parturient mothers. Since, it is a cost-effective, feasible, intervention without side effects this can be administered for all the mothers who are admitted to the labour room.

\section{References}

1. Anchana CK. Effectiveness of foot reflexology upon labour pain and coping in primigravid women (unpublished master's dissertation). Dr. MGR Medical University, Chennai 2013.

2. Baskar N. Textbook of Midwifery and obstetrical nursing (second edition). EMMESS medical publishers. 2015, P185.

3. Ebrahimzadeh S, Golmakani N, Kabirian M, Shakeri MT. Study of correlation between maternal fatigue and uterine contraction pattern in the active phase of labour. Journal of Clinical Nursing 2012;21(11-12):1563-1569. https://doi.org/10.1111/j.1365-2702.2012.04084.x

4. John E. A Comparative Study to Assess the Effectiveness of Selected Child Birth Preparation Methods. An Unpublished Doctoral Thesis submitted to 
Annamalai University, Chidambaram 2009.

5. Kinney MC. M Foundations of maternal - newborn and women's health nursing (5th edition). Saunders Elsevier publisher 2006, P422, 436 - 437.

6. Orshan SA. Maternity, Newborn, \& Health Nursing. Lippincott Williams and Wilkins 2008.

7. Pillitteri A, Pillitteri A. Maternal \& child health nursing: care of the childbearing \& childrearing family (6 $6^{\text {th }}$ edition). Wolters Kluwer 2010.

8. Pugh LC, Parks PL, Kitzrnan H. Clinical Approaches in the Assessment of Childbearing Fatigue 1999. https://www.jognn.org/article/S0884-2175(15)33615$7 / \mathrm{pdf}$

9. Synder M, Lindquist R. Complementary/alternative therapies in nursing. Springer publishing company 2006. 